\title{
Analytical Estimation of the Electrostatic Field in Cylinder-Plane and Cylinder-Cylinder Electrode Configurations
}

\author{
Emmanouil D. Fylladitakis ${ }^{1}$, Antonios X. Moronis ${ }^{2}$, Michael Theodoridis ${ }^{3}$ \\ ${ }^{1,3}$ Electronic \& Computer Engineering Department, Brunel University London, UK \\ ${ }^{2}$ Energy Technology Engineering Department, T.E.I. of Athens, Greece
}

\begin{tabular}{l} 
Article Info \\
\hline Article history: \\
Received Jul 5, 2016 \\
Revised Aug 13, 2016 \\
Accepted Aug 27, 2016 \\
\hline Keyword: \\
Cylinder-cylinder electrodes \\
Cylinder-plane electrodes \\
Electric field intensity \\
Electrostatic field \\
Equipotential lines \\
Field analysis
\end{tabular}

Corresponding Author:

Emmanouil D. Fylladitakis, Electronic \& Computer Engineering Department,

Brunel University London, Uxbridge, Middlesex, UK.

Email: emm.fyll@gmail.com

\begin{abstract}
This work presents analytical formulas for the estimation of the electrostatic field in cylinder-plane and cylinder-cylinder electrode configurations. Assuming a predefined potential difference between the electrodes and given their geometrical characteristics, these could be useful for the solution of numerous problems involving such electrode sets. Moreover, the voltage distribution around the electrodes is defined by providing equations either for the equipotentials at a given voltage ratio, or the exact estimation of the potential at any point in the surrounding space. Simplified expressions for critical engineering parameters such as the peak electric field and the field enhancement factor are also given.
\end{abstract}

Copyright $@ 2016$ Institute of Advanced Engineering and Science. All rights reserved.

\section{INTRODUCTION}

The electric field strength and the potential distribution are the key design parameters in a great number of electrical engineering applications, especially in dimensioning high voltage equipment [1-3]. The experimental measurement of the electric strength or voltage in the space surrounding voltage application electrodes, is usually difficult and inaccurate, due to the interference of the measuring devices with the electric field. Obviously, the introduction of analytical formulas for the field or the potential, becomes of great importance, though difficult to achieve in most cases. For that reason, the application of computer-aided simulations, based on finite element analysis are nowadays mostly preferable in most cases [4-6].

The aim of this study is the development of a mathematical model for the analytical calculation of the electric field strength and the potential distribution in wire - plane and parallel cylinder-cylinder electrode configurations of any dimensions. Such a mathematical model can be very useful in various real world applications, such as electrohydrodynamic (EHD) pump configurations, electrostatic precipitators, EHD dryers, electrostatic separators etc [5], [7-13]. Similar studies may be found in bibliography [14-16], nevertheless not providing full analytical solutions for the field strength, the potential distribution or the determination of the analytical equations of equipotentials all over the space surrounding the specific electrode sets under consideration. 


\section{RESEARCH METHOD}

It is known that the electric field distribution around two parallel cylindrical conductors of infinite length with identical cross-sections, can be calculated by assuming the presence of two equivalent line charges of opposite signs $+q_{l}$ and $-q_{l}$, each placed within a conductor. Due to longitudinal symmetry, the solution of the problem can be minimized in two dimensions, since the electric field is only depending on the distance perpendicular to the conductors, thus remaining unchanged along the longitudinal dimension. This model can be extended to solve cylinder to plane and twin cylinder to cylinder system configurations. In the case of a cylinder to plane configuration, the grounded plane can be assumed the plane of symmetry formed between two identical cylindrical conductors. Then, according to the theory of image charges [14], the problem can be solved by replacing the grounded plane with an equivalent negative image line charge $-q$ placed eccentrically behind the plane, at equal distance to the positive line charge $+q$ representing the cylindrical electrode, as shown in Figure 1.

Assuming air environment, the field intensity due to the presence of line charges can be found using the principle of superposition (Equation 1).

$$
P(x, y)=\frac{-q_{l}}{2 \pi \varepsilon_{0}} \ln \left(\frac{b}{a}\right)+Z
$$

where $Z$ is a constant defined from boundary conditions. For equal line charges of opposite polarity representing a wire-plane configuration, the potential at the plane should be zero (plane is grounded), therefore $Z=0$.

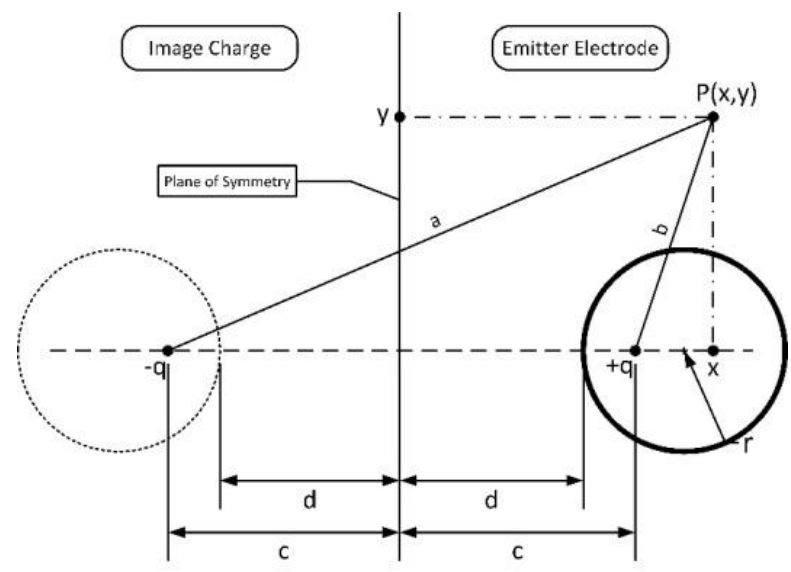

Figure 1. Geometrical Illustration of a Wire-to-plane Configuration Assuming the Formation of an Image Charge

On the other hand, in the case of twin cylindrical conductors, the plane of symmetry is at a voltage potential of $V / 2$, where $V$ is the voltage difference between the two electrodes. As such, the constant $Z$ equals $V / 2$.

The geometric parameters a and b can be easily found:

$$
\begin{aligned}
& a=\sqrt{(x+c)^{2}+y^{2}} \\
& b=\sqrt{(x-c)^{2}+y^{2}}
\end{aligned}
$$

It can be noticed that the formation of equipotential lines in any case is a set of circles, ranging from a pair of circles with radius $r$, representing the equipotential surface of each cylindrical conductor, to a circle of infinite radius, practically representing a line which coincides with the plane of symmetry. The centre of those circles is always on the $x$ axis. Therefore, the electric field intensity and the potential within the space surrounding the electrodes in the aforementioned configurations could be easily determined, provided the circle equations representing the equipotential lines are known. 


\section{MATHEMATICAL MODELS}

\subsection{Equipotential Lines}

Assuming that:

$$
A=\frac{-q_{l}}{2 \pi \varepsilon_{0}}
$$

and replacing Equation 2, 3, 4 into Equation 1, we have that, in the case of a wire-plane configuration $(Z=0)$, the equipotentials satisfy the condition:

$$
\ln \left(\frac{(x-c)^{2}+y^{2}}{(x+c)^{2}+y^{2}}\right)=\frac{2}{A} P_{e q}
$$

where $P_{e q}$ is a number that denotes different sets of equipotentials.

Rearranging Equation 5 we get:

$$
\left[x-\left(\frac{1+e^{\frac{2}{A} P_{e q}}}{1-e^{\frac{2}{A} P_{e q}}}\right) c\right]^{2}+y^{2}=c^{2}\left(\frac{1+e^{\frac{2}{A} P_{e q}}}{1-e^{\frac{2}{A} P_{e q}}}\right)^{2}-c^{2}
$$

The above equation shows that all equipotential lines are in fact circles, with centres lying on the $x$ axis. The abscissa of the centres of different equipotentials $x_{0}$ and the corresponding radius $R$ may are given as follows:

$$
\begin{aligned}
x_{0} & =\left(\frac{1+e^{\frac{2}{A} P_{e q}}}{1-e^{\frac{2}{A} P_{e q}}}\right) c \\
R^{2} & =c^{2}\left(\frac{1+e^{\frac{2}{A} P_{e q}}}{1-e^{\frac{2}{A} P_{e q}}}\right)^{2}-c^{2}=x_{0}{ }^{2}-c^{2}
\end{aligned}
$$

Equations 7 and 8 can be also written as:

$$
\begin{aligned}
& x_{0}=\operatorname{ccoth}\left(-\frac{P_{e q}}{A}\right) \\
& R=\sqrt{x_{0}^{2}-c^{2}}
\end{aligned}
$$

The above formulas define the centres and radii of equipotential lines provided the distance $\mathrm{c}$ between the plane of symmetry and the image line charges (Figure 1), as well as the magnitude q of the line charges, are both known.

The distance c can be easily found by considering the fact that the cylindrical conductor's surface is equipotential, thus satisfying equation 10 for $R=r$ and $x_{0}=d+r$. Accordingly, we get:

$$
c=\sqrt{d^{2}+2 d r}
$$

which defines $\mathrm{c}$ as a function of the already known geometrical parameters $\mathrm{d}$ and $\mathrm{r}$ (Figure 1).

Now the line charge magnitude $q$ needs also to be defined. This may be done by using Equation 1 for the electric potential at suitable points, $X_{1}(d, 0)$ and $X_{2}(-d, 0)$, on the surface of the cylindrical conductors of Figure 1, where the potential is already known ( $V$ and $-V$ respectively in the case of a wire-plane configuration where the grounded plane is equivalently substituted by the image charge $-q$ located inside the image cylinder at the left side of figure 1). Substituting in Equation 1 for $Z=0$ we finally get:

$$
P(X 1)=A \ln \left(\frac{\sqrt{(d-c)^{2}}}{\sqrt{(d+c)^{2}}}\right)=A \ln \left( \pm \frac{d-c}{d+c}\right)
$$


and

$$
P(X 2)=-A \ln \left(\frac{\sqrt{(-d-c)^{2}}}{\sqrt{(-d+c)^{2}}}\right)=-A \ln \left( \pm \frac{-d-c}{-d+c}\right)
$$

where:

$$
P(X 1)=-P(X 2)=V
$$

Combining Equation 14 with Equation 12 and Equation 13 results to four possible equation combinations. By solving them, it can be found that only one case yields an acceptable solution:

$$
V=A \ln \left(\frac{c-d}{c+d}\right)
$$

Substituting Equation 4 in Equation 15, we receive a solution for the charge $q_{l}$ in the case of a wirecylinder configuration, based only on known physical parameters.

$$
q_{l}=\frac{-2 \pi \varepsilon_{0} V}{\ln \left(\frac{c-d}{c+d}\right)}
$$

Similar methodology can be applied for the determination of equipotentials in the case of two parallel cylindrical conductors (wire-wire configuration) with potential difference $V$. In this case we have that $Z \neq 0$ in Equation 1, so Equation 5 takes the following form:

$$
\ln \left(\frac{(x-c)^{2}+y^{2}}{(x+c)^{2}+y^{2}}\right)=\frac{2}{A} \times\left(P_{e q}-Z\right)
$$

Therefore, Equation 9 now becomes:

$$
x_{0}=\operatorname{ccoth}\left(-\frac{P_{e q}-Z}{A}\right)
$$

In either case, the number $P_{e q}$ serves to specify the position of each equipotential circle. If one conductor is grounded, then the number $P_{e q}$ defines the equipotential circle at potential $V=P_{\text {Equation }}$ If the cylindrical conductors are at different potential with constant difference $V$, but neither of them is grounded, then the number Peq defines the equipotential circle at potential $V=P_{e q}+V_{\min }$ where $V_{\min }$ represents the lower potential between the two conductors. For $P_{e q}=V / 2$ the equipotential circle diminishes into a line positioned midway between the conductors $(R \rightarrow \infty)$.

Equation 10 and 11 for $R$ and $c$ are still valid, while further calculations determine that Equation 16 for the estimation of $q_{l}$ becomes:

$$
q_{l}=\frac{-\pi \varepsilon_{0} V}{\ln \left(\frac{c-d}{c+d}\right)}
$$

\subsection{Electric Field Analysis}

\subsubsection{Cylinder-Plane Configuration}

According to the analysis presented in the previous section and with reference to Figure 1, the electric potential for a cylinder-plane electrode set, at any point with Cartesian coordinates $(x, y)$, may be defined by combining Equation 1, Equation 2 and Equation 3 as follows:

$$
P_{c p}(x, y)=A_{c p} \ln \left(\frac{\sqrt{(x-c)^{2}+y^{2}}}{\sqrt{(x+c)^{2}+y^{2}}}\right)=\frac{A_{c p}}{2}\left[\ln \left((x-c)^{2}+y^{2}\right)-\ln \left((x+c)^{2}+y^{2}\right)\right]
$$

where $A_{c p}$ is derived by combining Equation 4 and Equation 15 : 
$A_{c p}=\frac{V}{\ln \left(\frac{c-d}{c+d}\right)}$

The corresponding electric field strength at point $(x, y)$ is defined as the gradient of potential:

$$
\boldsymbol{E}_{c \boldsymbol{p}}(\boldsymbol{x}, \boldsymbol{y})=-\nabla P_{c p}(x, y)=A_{c p}\left[\begin{array}{l}
{\left[\frac{-(x-c)}{(x-c)^{2}+y^{2}}+\frac{(x+c)}{(x+c)^{2}+y^{2}}\right] \boldsymbol{i}} \\
+\left[\frac{-y}{(x-c)^{2}+y^{2}}+\frac{y}{(x+c)^{2}+y^{2}}\right] \boldsymbol{j}
\end{array}\right]
$$

Combining Equation 21 and Equation 22 we finally get:

$$
E_{c p}(x, y)=-\frac{V}{\ln \frac{(c-d)}{(c+d)}} \frac{|2 c|}{\sqrt{c^{4}+2 c^{2}\left(y^{2}-x^{2}\right)+x^{4}+y^{4}}}
$$

Assuming that $y=0$ and $x=d$, then we get the peak electric field of the geometry:

$$
E_{\text {cpmax }}=-\frac{V}{\ln \frac{(c-d)}{(c+d)}} \times \frac{2 c}{c^{2}+d^{2}}
$$

Expanding, the Field Enhancement Factor can be calculated by dividing the average electric field of the geometry $\left(E_{a v g}=V / d\right)$ with the result of Equation 24. For inhomogeneous geometries that induce corona currents, the Field Enhancement Factor will always have a value lower than 1.

\subsubsection{Cylinder-Plane Configuration}

For a cylinder-cylinder configuration, Equation 20 takes the form:

$$
P_{c c}(x, y)=\frac{A_{c c}}{2}\left[\begin{array}{c}
\ln \left((x-c)^{2}+y^{2}\right) \\
-\ln \left((x+c)^{2}+y^{2}\right)
\end{array}\right]+Z
$$

where:

$$
A_{c c}=\frac{V}{2 \ln \left(\frac{c-d}{c+d}\right)}
$$

The corresponding electric field strength is defined as:

$$
E_{c c}(x, y)=-\frac{V}{\ln \frac{(c-d)}{(c+d)}} \frac{|c|}{\sqrt{c^{4}+2 c^{2}\left(y^{2}-x^{2}\right)+x^{4}+y^{4}}}
$$

\section{RESULTS AND DISCUSSION}

In order to verify the validity of the mathematical model, the results were compared to that derived via finite element analysis (FEA), which today is the most widely accepted computational method for solving similar problems. On that purpose, COMSOL Multiphysics $4.3 \mathrm{~b}$ has been used. Specifically, the simulations have been performed using the electrostatics module. In order to minimize any end-effects, the simulations were performed within a square boundary box of $80 \mathrm{~cm}$, with the cylindrical emitter facing a $60 \mathrm{~cm}$ wide plane collector. The maximum simulation distance between the electrodes is $5 \mathrm{~cm}$ and the emitter electrode is always at the exact centre of the boundary box. Table 1 displays the main software settings. Figure 2 displays the geometric representation of one of the FEA models that are being used to simulate the system.

To check the correlation between the results of the mathematical model and the FEA software output, three statistical functions are used. The first is the mean absolute percentage error (MAPE), described by Equation 28. 
$M A P E=\left[\frac{1}{n} \sum_{i=1}^{n}\left|\frac{E_{\text {sim }}-E_{e q}}{E_{\text {sim }}}\right|\right] \times 100 \%$

where $n$ is the number of data points, $E_{\text {sim }}$ is the simulated electric field strength and $E_{e q}$ is the calculated electric field strength using the mathematical model described in the previous sections.

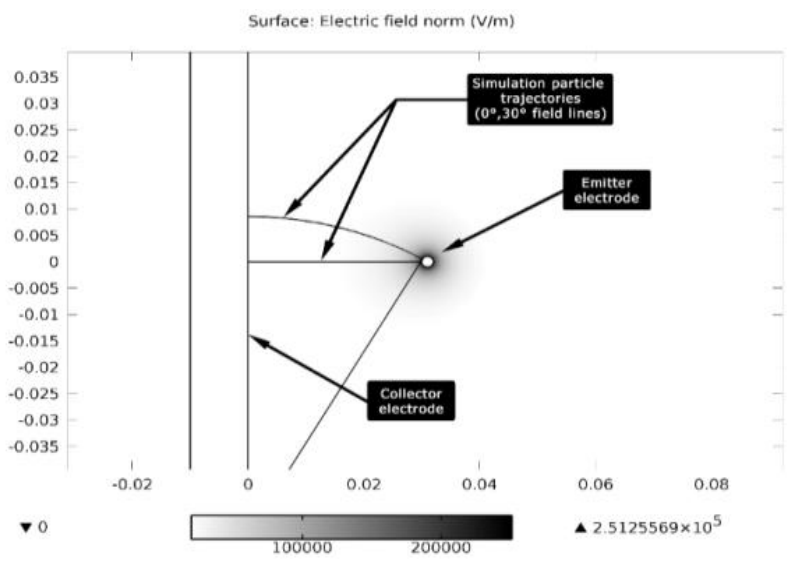

Figure 2. Output of a Demonstrative FEA Model, Displaying the Electric Field in the Space between a Cylinder-Plane Electrodes Set in Air. $(d=3 \mathrm{~cm}, r=1000 \mu \mathrm{m})$

The second statistical function is the mean absolute deviation (MAD), shown in Equation 29.

$$
M A D=\frac{1}{n} \sum_{i=1}^{n}\left|E_{\text {simi }}-E_{\text {eqi }}\right|
$$

The third statistical function is the weighted MAPE, also known as the MAD/Mean ratio, which assumes that the absolute error of each item is equally important [15]. Therefore, due to the very large scale of the electric field intensity, weighted MAPE is greatly affected by any errors very close to the top of the scale. The weighted MAPE is described by Equation 30.

$$
W M A P E=\left[\frac{\sum_{i=1}^{n}\left|E_{\text {simi }}-E_{\text {eqi }}\right|}{\sum_{i=1}^{n} E_{\text {simi }}}\right] \times 100 \%
$$

Multiple configurations of varying cylinder radius have been explored, as it can be seen from Table 1, which displays the MAD between the developed mathematical model and the results of the FEA software across the gap between the cylinder-plane electrode pair. Table 2 correspondingly displays the WMAPE error of the same configuration.

Table 1. COMSOL Multiphysics 4.3b Main Software Settings

$\begin{aligned} \text { Mesh element size on particle } & 1 \mu \mathrm{m} \text { maximum } \\ \text { trajectories } & \\ \text { Mesh element size in air domain } & 50 \mu \mathrm{m} \text { maximum } \\ \text { Resolution of curvatures } & 0.005 \\ \text { Maximum element growth rate } & 1.1 \\ \text { Resolution of narrow regions } & 0.01 \\ \text { Solver type } & \text { Stationary, MUMPS } \\ \text { Solver relative tolerance } & 0.001 \\ \text { Pivot Threshold } & 0.1\end{aligned}$


Table 2. Electric Field Gradient MAD between the Mathematical and Simulated Models, Cylinder-Plane Configuration, $\varphi=0^{\circ}$

\begin{tabular}{ccccc}
\hline \multirow{2}{*}{$d(\mathrm{~cm})$} & 50 & 500 & 1000 & 5000 \\
\hline 1 & $46.87 \mathrm{~V} / \mathrm{m}$ & $19.92 \mathrm{~V} / \mathrm{m}$ & $17.85 \mathrm{~V} / \mathrm{m}$ & $8.09 \mathrm{~V} / \mathrm{m}$ \\
2 & $46.63 \mathrm{~V} / \mathrm{m}$ & $39.81 \mathrm{~V} / \mathrm{m}$ & $12.75 \mathrm{~V} / \mathrm{m}$ & $25.96 \mathrm{~V} / \mathrm{m}$ \\
3 & $59.76 \mathrm{~V} / \mathrm{m}$ & $59.60 \mathrm{~V} / \mathrm{m}$ & $58.52 \mathrm{~V} / \mathrm{m}$ & $45.72 \mathrm{~V} / \mathrm{m}$ \\
5 & $92.60 \mathrm{~V} / \mathrm{m}$ & $97.45 \mathrm{~V} / \mathrm{m}$ & $97.54 \mathrm{~V} / \mathrm{m}$ & $85.82 \mathrm{~V} / \mathrm{m}$ \\
\hline
\end{tabular}

To verify the validity of the mathematical model, its results across other field lines were examined as well. Using the particle tracing module present in COMSOL Multiphysics, the trajectory of a particle leaving an emission angle $\varphi$ (Figure 3 ) is recorded and the results across that trajectory are being compared to that derived using the mathematical model. This trajectory coincides with the field line for an emission angle $\varphi$, which is perpendicular to both the surface of the emitter and the plane of symmetry [18].

Similarly as before, Tables 3 and 4 respectively display the MAD and the WMAPE between the presented mathematical model and the results of the FEA software across the field line for an emission angle of $30^{\circ}$.

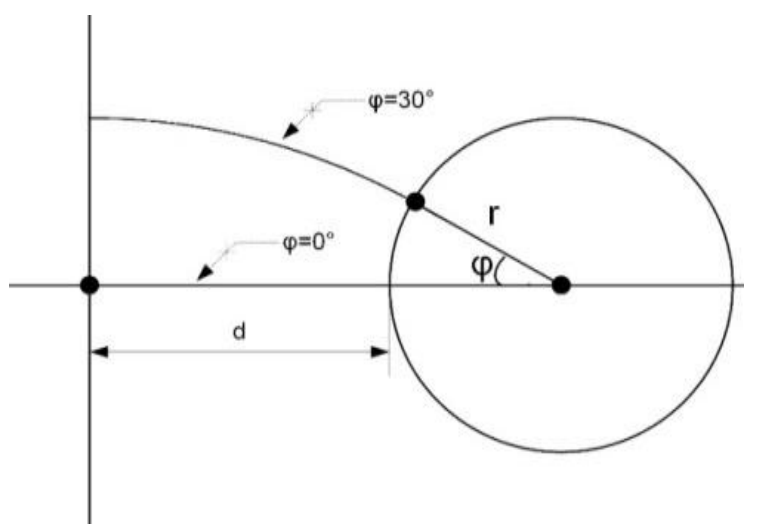

Figure 3. Particle Trajectories (Field Lines) for Emission Angles $\varphi=0^{\circ}$ and $\varphi=30^{\circ}$

Table 3. Electric Field Gradient WMAPE between the Mathematical and Simulated Models, Cylinder-Plane

\begin{tabular}{ccccc}
\multicolumn{5}{c}{ Configuration, $\varphi=0^{\circ}$} \\
\hline \multirow{2}{*}{$d(\mathrm{~cm})$} & 50 & 500 & 1000 & 5000 \\
\hline 1 & $0.046 \%$ & $0.020 \%$ & $0.018 \%$ & $0.008 \%$ \\
2 & $0.091 \%$ & $0.079 \%$ & $0.025 \%$ & $0.052 \%$ \\
3 & $0.176 \%$ & $0.178 \%$ & $0.175 \%$ & $0.137 \%$ \\
5 & $0.454 \%$ & $0.486 \%$ & $0.487 \%$ & $0.429 \%$ \\
\hline
\end{tabular}

Table 4. Electric Field Gradient MAD between the Mathematical and Simulated Models, Cylinder-Plane Configuration, $\varphi=30^{\circ}$

\begin{tabular}{ccccc}
\hline \multirow{2}{*}{$d(\mathrm{~cm})$} & 50 & 500 & 1000 & 5000 \\
\hline 1 & $58.18 \mathrm{~V} / \mathrm{m}$ & $21.54 \mathrm{~V} / \mathrm{m}$ & $19.82 \mathrm{~V} / \mathrm{m}$ & $14.08 \mathrm{~V} / \mathrm{m}$ \\
2 & $53.19 \mathrm{~V} / \mathrm{m}$ & $40.74 \mathrm{~V} / \mathrm{m}$ & $40.17 \mathrm{~V} / \mathrm{m}$ & $33.62 \mathrm{~V} / \mathrm{m}$ \\
3 & $63.56 \mathrm{~V} / \mathrm{m}$ & $60.32 \mathrm{~V} / \mathrm{m}$ & $60.45 \mathrm{~V} / \mathrm{m}$ & $54.13 \mathrm{~V} / \mathrm{m}$ \\
5 & $91.31 \mathrm{~V} / \mathrm{m}$ & $32.60 \mathrm{~V} / \mathrm{m}$ & $99.31 \mathrm{~V} / \mathrm{m}$ & $95.01 \mathrm{~V} / \mathrm{m}$ \\
\hline
\end{tabular}

Furthermore, simulations were run for cylinder-cylinder configurations as well. Tables 5, 6, and 7 respectively display the MAD and the WMAPE between the presented mathematical model and the results of the FEA software across the field line for an emission angle of $0^{\circ}$, for a cylinder-tocylinder configuration. The distance $d$ is the distance of each electrode from the plane of symmetry. 
The MAPE error of all configurations is deliberately omitted, as it was entirely insignificant for every configuration that has been tested.

Table 5. Electric Field Gradient WMAPE between the Mathematical and Simulated Models, Cylinder-Plane Configuration, $\varphi=30^{\circ}$

\begin{tabular}{ccccc}
\hline \multirow{2}{*}{$d(\mathrm{~cm})$} & 50 & 500 & 1000 & 5000 \\
\hline 1 & $0.060 \%$ & $0.023 \%$ & $0.021 \%$ & $0.016 \%$ \\
2 & $0.111 \%$ & $0.086 \%$ & $0.085 \%$ & $0.073 \%$ \\
3 & $0.198 \%$ & $0.190 \%$ & $0.191 \%$ & $0.174 \%$ \\
5 & $0.475 \%$ & $0.513 \%$ & $0.521 \%$ & $0.504 \%$ \\
\hline
\end{tabular}

Table 6. Electric Field Gradient MAD between the Mathematical and Simulated Models, Cylinder-Cylinder Configuration, $\varphi=0^{\circ}$

\begin{tabular}{ccccc}
\hline \multirow{2}{*}{$d(\mathrm{~cm})$} & 50 & 500 & 1000 & 5000 \\
\hline 1 & $18.20 \mathrm{~V} / \mathrm{m}$ & $2.51 \mathrm{~V} / \mathrm{m}$ & $2.14 \mathrm{~V} / \mathrm{m}$ & $0.91 \mathrm{~V} / \mathrm{m}$ \\
2 & $10.54 \mathrm{~V} / \mathrm{m}$ & $4.63 \mathrm{~V} / \mathrm{m}$ & $1.46 \mathrm{~V} / \mathrm{m}$ & $2.93 \mathrm{~V} / \mathrm{m}$ \\
3 & $9.62 \mathrm{~V} / \mathrm{m}$ & $6.88 \mathrm{~V} / \mathrm{m}$ & $6.72 \mathrm{~V} / \mathrm{m}$ & $5.20 \mathrm{~V} / \mathrm{m}$ \\
5 & $11.62 \mathrm{~V} / \mathrm{m}$ & $11.36 \mathrm{~V} / \mathrm{m}$ & $11.34 \mathrm{~V} / \mathrm{m}$ & $9.88 \mathrm{~V} / \mathrm{m}$ \\
\hline
\end{tabular}

Table 7. Electric Field Gradient WMAPE between the Mathematical and Simulated Models, Cylinder-Cylinder Configuration, $\varphi=0^{\circ}$

\begin{tabular}{ccccc}
\hline \multirow{2}{*}{$d(\mathrm{~cm})$} & 50 & 500 & 1000 & 5000 \\
\hline 1 & $0.036 \%$ & $0.005 \%$ & $0.004 \%$ & $0.002 \%$ \\
2 & $0.042 \%$ & $0.018 \%$ & $0.006 \%$ & $0.012 \%$ \\
3 & $0.057 \%$ & $0.041 \%$ & $0.040 \%$ & $0.031 \%$ \\
5 & $0.116 \%$ & $0.114 \%$ & $0.113 \%$ & $0.099 \%$ \\
\hline
\end{tabular}

All of the calculated errors are minimal and caused by the computing capabilities of the software performing the calculations. For example, decreasing the solver's tolerance and or reducing the mesh element size in COMSOL Multiphysics increases the convergence between the mathematical model and the simulation results even further, yet at the substantial expense of computing time. In addition to the above, Microsoft Excel is being used for the calculation of the mathematical model. Using an arbitrary precision arithmetic calculator for the mathematical functions may increase the accuracy of the mathematical results even further, yet they will not coincide with the results of a FEA model perfectly, which are also limited by the capabilities of the software.

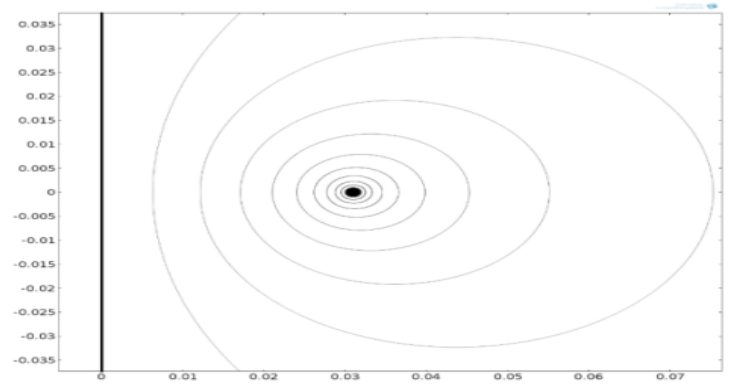

Figure 4. Formation of Equipotential Lines Surrounding a Cylindrical Electrode Facing a Grounded Plane Electrode, for $V=1 \mathrm{kV}, d=3 \mathrm{~cm}$ and $r=0.5 \mathrm{~cm}$, in $100 \mathrm{~V}$ steps

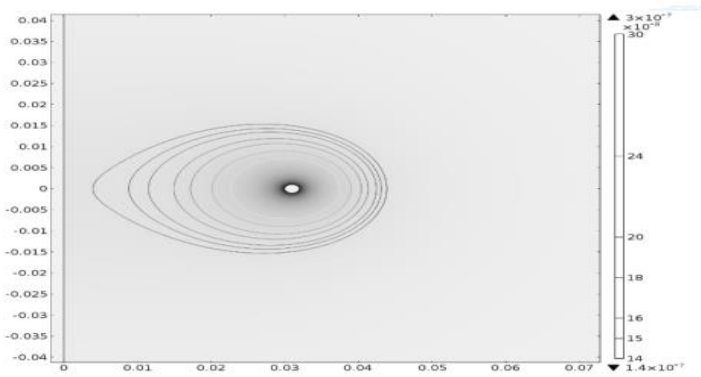

Figure 5. Electric Field Surrounding a Cylindrical Electrode Facing a Grounded Plane Electrode, for $\mathrm{V}=1 \mathrm{kV}, \mathrm{d}=3 \mathrm{~cm}$ and $\mathrm{r}=0.5 \mathrm{~cm}$

As mentioned in the previous section of this paper, near the end of the equipotential modelling paragraph, valid $P_{e q}$ figures range from zero to $V$. Each number $P_{e q}$ describes a specific equipotential line, the 
electric potential of which is equal to $P_{\text {Equation }}$ Therefore, if the radius $r$ of the emitter electrode, the distance $d$ from the emitter electrode to the plane of symmetry and the potential $V$ applied to the emitter electrode are all known, the exact formation of the equipotential lines is known as well. Figure 4 illustrates the formation of equipotential lines of a cylinder-plane configuration for $V=1 \mathrm{kV}, d=5 \mathrm{~cm}$ and $r=0.5 \mathrm{~cm}$, with a $P_{e q}$ from 100 to 900 , in steps of 100 . These results were also successfully validated by comparing the output of the mathematical model to that of the FEA software as shown in Figure 5.

\section{CONCLUSION}

This paper presented a mathematical model for the analytical calculation of the electric field and potential formed between cylinder-plane or parallel cylinder-cylinder configurations. Computer assisted simulations were used to verify the validity of the mathematical model, with excellent results, proving its accuracy. The developed mathematical model has numerous practical applications, small and large scale alike. It can be used to calculate the electric field surrounding wire-plane or parallel wire configurations of any dimensions. Such geometries are present in a variety of devices, from electrohydrodynamic pumps to electrostatic precipitators and ion generators. It also may be used to estimate the potential at any point in the space surrounding these configurations by finding the exact equipotential line that crosses that point. These parameters are quite important in engineering design, such as for proper calculation of insulation and safety measures.

\section{REFERENCES}

[1] M. Khalifa, High Voltage Engineering: Theory and Practice. New York: Marcel and Dekker, 1990.

[2] M.S. Naidu and V. Kamaraju, High voltage engineering. New York: Tata McGraw-Hill Education, 2013.

[3] C. Wadhwa, High voltage engineering. New Delhi: New Age International, 2007.

[4] L. Yanju, C. Yundong, L. Yanbin, G. Youhua, and L. Xiaoming, "Design for new type of main insulation of 35kV electric power transformer based on electric field analysis", in Automation Congress, 2008. WAC 2008. World, 2008; 1: 1-4.

[5] E.D. Fylladitakis, M.P. Theodoridis, and A.X. Moronis, "Review on the History, Research, and Applications of Electrohydrodynamics", Plasma Science, IEEE Transactions on, 2014; 42: 358-375.

[6] D. Meeker, "Finite Element Method Magnetics (FEMM), Version 4.2", Web Page: http://femm.berlios.de, 2006.

[7] J.C. Matéo-Vélez, P. Degond, F. Rogier, A. Séraudie, and F. Thive, "Modelling wire-to-wire corona discharge action on aerodynamics and comparison with experiment", J. Phys. D: Appl. Phys., 2008; 41: 1-11.

[8] P. Glushchenko and Y.K. Stishkov, "Modeling of the through EHD-flow structure in a wire-wire system", Surface Engineering and Applied Electrochemistry, 2007; 43: 257-264.

[9] L. Zhao, E.D. Cruz, K. Adamiak, A. Berezin, and J. Chang, "A numerical model of a wire-plate electrostatic precipitator under electrohydrodynamic flow conditions", in CD-ROM proceedings of the international conference on air pollution abatement technologies-Future challenges, Cairns, Australia, 2006.

[10] F. Lai and K.W. Lai, "EHD-enhanced drying with wire electrode", Drying Technology, July 2002; 20: 1393-1405.

[11] S. Kurokawa, J.P. Filho, M.C. Tavares, C.M. Portela, and A.J. Prado, "Behavior of overhead transmission line parameters on the presence of ground wires", Power Delivery, IEEE Transactions on, 2005; 20: 1669-1676.

[12] A. Ma, D. Xu, Z. Zhang, F. Li, and L. Zhao, "The nominal field strength calculation of UHVDC transmission lines based on surface charge method", in Power System Technology (POWERCON), 2014 International Conference on, 2014; 1: 2242-2246.

[13] A.I. Sidorov, I.S. Okrainskaya, and S.P. Gladyshev, "Measurement of super high voltage transmission line electric field effecting on the environment", in Electro/Information Technology (EIT), 2011 IEEE International Conference on, 2011; 1: 1-4.

[14] J. Kuffel, E. Kuffel, and W. Zaengl, High voltage engineering fundamentals: Newnes, 2000.

[15] K.R.K. Rajagopala, K.P. Vittal, and H. Lunavath, "Computation of electric field and thermal properties of 3-phase cable", TELKOMNIKA (Telecommunication Computing Electronics and Control), 2012; 10: 265-274.

[16] S. Mpanga, W. Feng, and C. Chun, "Electromagnetic Field Evaluation of a 500kV High Voltage Overhead Line", Indonesian Journal of Electrical Engineering and Computer Science, 2013; 11: 789-796.

[17] S. Kolassa and W. Schütz, "Advantages of the MAD/MEAN ratio over the MAPE", Foresight: The International Journal of Applied Forecasting, 2007; 1: 40-43.

[18] A.X. Moronis, N. Simou, K.N. Kiousis, and E.D. Fylladitakis, "A model for determining the unipolar ionic saturation current in parallel wire-cylinder electrodes during corona discharge", Dielectrics and Electrical Insulation, IEEE Transactions on, 2014; 21: 1035-1043. 


\section{BIOGRAPHIES OF AUTHORS}
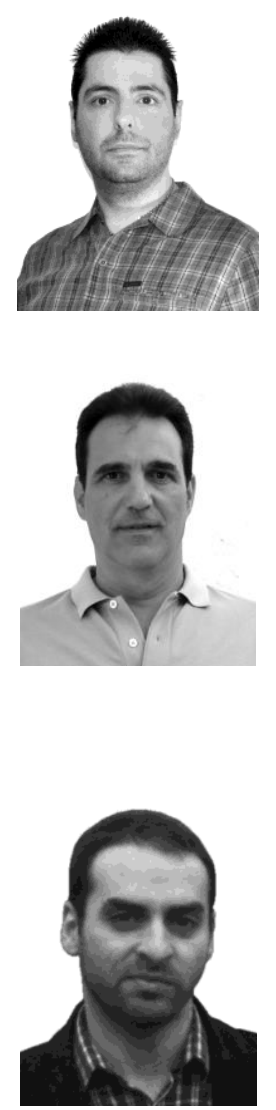

Emmanouil D. Fylladitakis received a B.Sc degree in electrical energy engineering from the Technological Educational Institute (TEI) of Athens, Athens, Greece, in 2010, an M.Sc degree in energy with distinction from the Heriot-Watt University, Edinburgh, Scotland, in 2012, where he received a prize for outstanding merit, and a Ph.D degree from the Electronics and Computer Engineering department of the Brunel University London, U.K, in 2016. His research interests include the study of corona discharges, electrohydrodynamic effects, renewable energy systems, energy conservation in buildings, engineering education and distance learning systems. He currently is an external research associate at Brunel University London and the Technological Educational Institute (TEI) of Athens.

Antonios X. Moronis was born in Athens, Greece, in 1967. He received a diploma in electrical engineering from the Aristotle University of Thessaloniki, Greece, in 1990, and a PhD degree in electrical engineering from the National Technical University of Athens in 1995. From 2001 to 2006 he was Assistant Professor with the Energy Engineering Department of Technological Educational Institute, Athens, Greece. Currently he is an Associate Professor at the same Department and director of the Electrotechnology and Measuring Systems laboratory. He is the author of more than 45 articles in scientific journals and conference proceedings. His research interests include dielectrics and electrical insulation, electrohydrodynamics, high voltage applications, earthing systems in electrical installations, and fault diagnosis and predictive maintenance of electric power system components.

Dr. Moronis is a member of the Technical Chamber of Greece.

Michael P. Theodoridis received the B.Sc. degree in energy engineering from the Technological Educational Institute (TEI) of Athens, Athens, Greece, in 2000, the M.Sc. degree in power electronics and drives jointly from the University of Birmingham, Birmingham, U.K., and the University of Nottingham, Nottingham, U.K., in 2002, and the Ph.D. degree in electrical engineering from the University of Birmingham in 2005. From 2000 to 2001, he was at Olympic Airways, Athens, Greece. From 2005 to 2011, he was with the Department of Energy Technology, TEI of Athens. He is currently with Brunel University London, U.K. His research interests include high-frequency converters, electrical machines and drives, and renewable energy. 